RESEARCH ARTICLE

\title{
Food Addiction in a Spanish Sample of Eating Disorders: DSM-5 Diagnostic Subtype Differentiation and Validation Data
}

\author{
Roser Granero ${ }^{1,2 \dagger}$, Ines Hilker ${ }^{3 \dagger}$, Zaida Agüera², Susana Jiménez-Murcia ${ }^{2,3,4}$, Sarah Sauchelli ${ }^{3}$, \\ Mohammed A. Islam², Ana B. Fagundo ${ }^{2,3}$, Isabel Sánchez ${ }^{3}$, Nadine Riesco $^{3}$, Carlos Dieguez ${ }^{2,5}$, José Soriano ${ }^{6}$, \\ Cristina Salcedo-Sánchez ${ }^{6}$, Felipe F. Casanueva ${ }^{2,7}$, Rafael De la Torre ${ }^{2,8}$, José M. Menchón ${ }^{3,4,9}$, \\ Ashley N. Gearhardt ${ }^{10}$ \& Fernando Fernández-Aranda ${ }^{2,3,4 *}$ \\ ${ }^{1}$ Departament de Psicobiologia i Metodologia de les Ciències de la Salut, Universitat Autònoma de Barcelona, Barcelona, Spain \\ ${ }^{2}$ CIBER Fisiopatologia Obesidad y Nutrición (CIBERObn), Instituto de Salud Carlos III, Madrid, Spain \\ ${ }^{3}$ Department of Psychiatry, Bellvitge University Hospital-IDIBELL, Barcelona, Spain \\ ${ }^{4}$ Clinical Sciences Department, School of Medicine, Barcelona, Spain \\ ${ }^{5}$ Department of Physiology, CIMUS, University of Santiago de Compostela-Instituto de Investigación Sanitaria, Santiago de Compostela, Spain \\ ${ }^{6}$ Department of Psychiatry, University Hospital of Sant Pau, Barcelona, Spain \\ ${ }^{7}$ Laboratory of Molecular and Cellular Endocrinology, Research Area, Complejo Hospitalario Universitario de Santiago de Compostela, A Coruña, Spain \\ ${ }^{8}$ Human Pharmacology and Clinical Neurosciences Research Group, Neuroscience Research Program, IMIM (Hospital del Mar Medical Research Institute), Barcelona, Spain \\ ${ }^{9}$ CIBER Salud Mental (CIBERSAM), Instituto Salud Carlos III, Barcelona, Spain \\ ${ }^{10}$ Department of Psychology, University of Michigan, Ann Arbor, MI, USA
}

\begin{abstract}
Although the concept of 'food addiction' (FA) has raised growing interest because of evidence for similarities between substance dependence and excessive food intake, there is a lack of studies that explore this construct among the wide spectrum of eating disorders (EDs). Besides providing validation scores of a Spanish version of the Yale FA Scale (YFAS-S), this study examined the prevalence of 'FA' among ED subtypes compared with healthy-eating controls (HCs) and the association between 'FA' scores, eating symptomatology and general psychopathology. A sample of 125 adult women with ED, diagnosed according to Diagnostic and Statistical Manual of Mental Disorders 5 criteria, and 82 healthy-eating women participated in the study. All participants were assessed with the YFAS-S, the ED Inventory-2 and the Symptom Checklist-Revised. Results showed that the internal structure of the one-dimensional solution for the YFAS-S was very good $(\alpha=0.95)$. The YFAS-S has a good discriminative capacity to differentiate between ED and controls (specificity=97.6\% and sensitivity $(\mathrm{Se})=72.8 \%$; area under receiver operating characteristic curve $=0.90)$ and a good Se to screen for specific ED subtypes. YFAS-S scores were associated with higher levels of negative affect and depression, higher general psychopathology, more severe eating pathology and greater body mass index. When comparing the prevalence of 'FA' between ED subtypes, the lowest prevalence of 'FA', measured with the YFAS-S, was for the anorexia nervosa (AN) restrictive subtype with 50\%, and the highest was for the AN binge-purging subtype (85.7\%), followed by bulimia nervosa $(81.5 \%)$ and binge eating disorder (76.9\%). In conclusion, higher YFAS-S scores are associated with bingeing ED-subtype patients and with more eating severity and psychopathology. Although the 'FA' construct is able to differentiate between ED and HC, it needs to be further explored. Copyright (c) 2014 John Wiley \& Sons, Ltd and Eating Disorders Association.
\end{abstract}

Keywords

eating disorders; anorexia nervosa; bulimia nervosa; binge eating disorder; food addiction; YFAS

\section{*Correspondence}

Fernando Fernández-Aranda, PhD, Head of Eating Disorders Unit, Department of Psychiatry, Bellvitge University Hospital-IDIBELL and CIBERobn, c/ Feixa Llarga s/n, 08907 L'Hospitalet de Llobregat, Barcelona, Spain. Tel: +34 9326079 88; Fax: +34 932607658.

Email: ffernandez@bellvitgehospital.cat

${ }^{\dagger}$ These authors have shared first authorship.

Published online 20 August 2014 in Wiley Online Library (wileyonlinelibrary.com) DOI: 10.1002/erv.2311

\section{Introduction}

Interest in the concept of 'food addiction' (FA) is growing steadily, and there is more and more evidence of similarities between substance dependence and excessive food intake. Research with animal models supports the possibility of the development of addictive-like behaviours to some food components especially to food high in sugar and fat (Nogueiras et al., 2012). In rats that binged on sugar, neurochemical signs of dependence (e.g. changes in dopamine and opioid receptor binding and repeated dopamine and acetylcholine release in the nucleus accumbens) as well as behavioural signs (e.g. increased intake after abstinence and cross 
sensitization with drugs of abuse) have been observed (Avena, Bocarsly, \& Hoebel, 2012; Avena, Rada, \& Hoebel, 2008) although in most instances, they do not become obese. Johnson and Kenny demonstrated that the overconsumption of palatable high-fat food triggered neuroadaptative responses in brain reward circuits in obese rats that were similar to those seen in substance dependence and that led to a pattern of compulsive eating (Johnson \& Kenny, 2010), although no signs of opiate-like withdrawal in fat-bingeing rats were detected (Avena, 2010).

Findings from neuroimaging studies suggest that similar neuronal circuits, modulated by dopamine, are activated in addiction and obesity by either drugs or hyperpalatable food (Volkow, Wang, Fowler, \& Telang, 2008; Volkow, Wang, Fowler, Tomasi, \& Baler, 2012). Wang, Volkow, Thanos, and Fowler (2009) identified that in both conditions, there is a decreased availability of dopamine D2 receptors and a decreased metabolism in the prefrontal regions involved in inhibitory control. Furthermore, other authors (Gearhardt et al., 2011) observed that in a sample of lean to obese young women, higher scores on the Yale FA Scale (YFAS) were associated with similar patterns of neural activation to that of substance dependence, such as elevated activation of the reward circuitry in response to food cues and reduced activation of inhibitory regions in response to food intake. Although there are some behavioural and neurobiological pieces of evidence supporting a food addiction phenotype in humans, its validity is still controversial (Hone-Blanchet \& Fecteau, 2014; Ziauddeen \& Fletcher, 2013).

Several studies have explored the possibility of 'FA' in humans. Some of the first authors to examine the prevalence of ' $F A$ ' in a sample of women with binge eating disorder (BED) found that 92.4\% met the Diagnostic and Statistical Manual of Mental Disorders, fourth edition, (DSM-IV) criteria modified for substance dependence, although the percentage was lower when using more restrictive criteria (Cassin \& von Ranson, 2007). Gearhardt and colleagues (Gearhardt, Corbin, \& Brownell, 2009a) examined the diagnostic criteria for food dependence and other psychological characteristics of addiction to develop the YFAS, the first validated tool to identify individuals with addictive behaviours towards food in accordance with substance dependence criteria. The YFAS was initially validated with a non-clinical sample (Gearhardt, Corbin, \& Brownell, 2009b), which showed adequate reliability and validity, and was later assessed with samples of obese adults (Gearhardt et al., 2012) and children (Gearhardt, Roberto, Seamans, Corbin, \& Brownell, 2013).

Some authors suggested that 'FA' could be a valid phenotype of obesity, as in their sample of obese adults, 25\% met diagnostic criteria for 'FA' when assessed using the YFAS (Davis et al., 2011). Similarly, in a sample of individuals seeking weight loss treatment, $15 \%$ met the YFAS criteria for 'FA' (Eichen, Lent, Goldbacher, \& Foster, 2013), and in two different samples of bariatric surgery individuals, 41.7\% (Meule, Heckel, \& Kübler, 2012) and $53.7 \%$ (Clark \& Saules, 2013) met the criteria for 'FA'. Finally, in samples of obese adults with BED, 57\% of predominantly Caucasian individuals were classified as meeting 'FA' criteria (Gearhardt et al., 2012), whereas in a racially diverse sample, the percentage was of 41.5. Moreover, patients with a diagnosis of BED who meet the YFAS criteria for 'FA' present more ED pathology and associated psychopathology (Gearhardt et al., 2012; Gearhardt, White, Masheb, \& Grilo, 2013). This is specifically related to dopaminergic activation (Davis et al., 2013) and to disinhibited eating and food craving (Burgess, Turan, Lokken, Morse, \& Boggiano, 2014).

After considering the existing literature on comorbidity between EDs and other substance and non-substance-related disorders (Calero-Elvira et al., 2009; Jiménez-Murcia et al., 2013; Speranza et al., 2012), we hypothesized that a similar 'FA' pattern would be observed in other subtypes of ED, particularly bulimia nervosa (BN). To our knowledge, there are no studies that have explored the prevalence of 'FA' in other EDs apart from BED and BN, namely anorexia nervosa (AN), or ED not otherwise specified (EDNOS). Neither is there research conducted with clinical populations that are not of Anglo-Saxon origins, which questions the applicability of the construct of 'FA' in other cultures.

\section{Aims}

The aims of our study were fourfold: (i) to translate the YFAS (Gearhardt et al., 2009b) into Spanish and provide validation scores of the YFAS-Spanish (YFAS-S) using a clinical sample of adults with ED; (ii) to examine the prevalence of 'FA' and assess differences in YFAS-S dependence criteria in ED patients compared with healthy controls (HCs); (iii) to explore differences between ED subtypes in 'FA' and among the YFAS-S dependence criteria; and (iv) to examine the associations between YFAS-S scoring, eating symptomatology and general psychopathology.

\section{Method}

\section{Participants}

Entry into the study was between September 2013 and March 2014. The final sample included 207 participants [125 female ED patients (40 AN, $54 \mathrm{BN}, 18$ EDNOS and $13 \mathrm{BED}$ ) and 82 healthy female controls (HCs)]. All ED patients were consecutively admitted to the ED Units at the University Hospital of Bellvitge and the Hospital de la Santa Creu i Sant Pau in Barcelona, and all controls were from the same catchment area as index patients. ED diagnoses were originally made according to DSM-IV Text Revision (APA, 2000) criteria, using a semistructured clinical interview (Structured Clinical Interview for DSM-IV Axis I disorders; First, Gibbon, Spitzer, \& Williams, 1996) conducted by experienced psychologists and psychiatrists. These diagnoses were reanalysed post hoc using the recent DSM-5 criteria (APA, 2013), as described recently (Machado, Goncalves, \& Hoek, 2013). The ED sample had a mean age of 29.3 years $(S D=10.9)$, a mean age at onset of 19.0 years $(S D=7.6)$ and a mean body mass index $\left(\mathrm{BMI}, \mathrm{kg} / \mathrm{m}^{2}\right)$ of $23.2(S D=7.5)$, and the mean duration of the ED was 9.5 years $(S D=9.5$; median 5 years). The HC sample comprised women who were visiting the hospital for routine blood tests and students who volunteered. Mean age was 23.4 years old $(S D=5.3)$, and mean BMI was $21.3 \mathrm{~kg} / \mathrm{m}^{2}$ $(S D=3.0)$.

Patients were excluded from the analyses if they had missing values for any diagnostic item. For the present analysis, from an initial sample of $139 \mathrm{ED}$ patients, the following individuals were excluded: males $(n=3)$, as the number of men with ED diagnosis was too small for a meaningful comparison, and 11 patients with missing relevant data. Exclusion criteria for the HC group were 
being male, having a lifetime diagnosis of ED or a current diagnosis of any substance or non-substance abuse disorder. The assessment of a lifetime history of health or mental illness was based on the general health questionnaire (General Health Questionnaire 28) (Goldberg \& Hillier, 1979; Lobo, Pérez-Echeverría, \& Artal, 2009). None of them were taking any medication.

The Ethics Committee of our institution (Ethics Committee of Clinical Research of the Bellvitge University Hospital) approved this study, and written informed consent was obtained from all participants.

\section{Assessment}

For the assessment, commonly applied questionnaires in the field of EDs, comprising the ED Inventory-2 (EDI-2) and the Symptom Checklist-Revised (SCL-90-R) (Derogatis, 1990), were administered. Additionally, the YFAS was used for measuring food addiction construct (Gearhardt et al., 2009b). Furthermore, information concerning clinical variables was assessed by means of a semistructured clinical interview (Fernandez-Aranda \& Turon, 1998).

\section{Yale Food Addiction Scale-Spanish (adapted from Gearhardt et al., 2009b)}

The YFAS measures symptoms of food addiction, and its Spanish translation version was used in the current study. This 25 -item selfreport questionnaire was designed to measure food addiction according to the seven symptoms of substance dependence listed in the DSM-IV (e.g. withdrawal, tolerance, continued use despite problems, vulnerability in social activities, etc.), modified for eating behaviours. This instrument uses two scoring options to indicate the experience of addictive eating behaviour; a dimensional score (Likert scale) and a binary score. 'FA' is diagnosed when at least three symptoms and a clinically significant impairment or distress (as assessed with two extra items) are present within the previous 12 months. The validation of the English version shows an adequate internal reliability and good convergent and incremental validity in predicting binge eating (Gearhardt et al., 2009b).

The most common method of translation used for crosscultural research includes back-translation, consultation with bilingual individuals who review the translation, and pre-testing the translated measure for further revisions (Brislin, 1970). Both linguistic and cultural differences influence the process of translation into another language. In the translation and back-translation processes, linguistic and grammatical aspects are taken into account, as well as possible cultural differences. Two translators that are specialists in the field translated the YFAS from English into Spanish. After, a third translator corrected the linguistic and grammatical errors in the Spanish version. Finally, a fourth translator translated the Spanish version back into English. A copy of the Spanish translation can be obtained from the last author (Dr Fernando Fernández-Aranda, ffernandez@bellvitgehospital.cat or from Ashley Gearhardt, agearhar@umich.edu).

\section{Eating Disorder Inventory-2 (Garner, 1991)}

This is a reliable and valid 91-item multidimensional self-report questionnaire that assesses different cognitive and behavioural characteristics, which are typical for ED. The EDI-2 retains the 64 items (grouped into eight scales: drive for thinness, bulimia, body dissatisfaction, ineffectiveness, perfectionism, interpersonal distrust, interoceptive awareness, and maturity fears) of the EDI and adds 27 new items into three provisional scales: Asceticism, impulse regulation, and social insecurity. All of these scales are answered on a six-point Likert scale and provide standardized subscale scores. This instrument was validated in a Spanish population (Garner, 1998 ) with a moderate mean internal consistency of $\alpha=0.63$.

\section{Symptom Checklist-Revised ( Derogatis, 1990)}

This test contains 90 items and helps measure nine primary symptom dimensions of psychopathology, which are as follows: (i) somatisation; (ii) obsession-compulsion; (iii) interpersonal sensitivity (Se); (iv) depression; (v) anxiety; (vi) hostility; (vii) phobic anxiety; (viii) paranoid ideation; and (ix) psychoticism. In addition, it includes three global indices, which are a global severity index (GSI), designed to measure overall psychological distress, a positive symptom distress index (PSDI), designed to measure the intensity of symptoms, and a positive symptom total (PST), which measures self-reported symptoms. The GSI can be used as a summary of the test. This scale has been validated in a Spanish population (Derogatis, 2002), obtaining a mean internal consistency of 0.75 (coefficient alpha).

\section{Statistical analysis}

Confirmatory factor analysis (CFA) with STATA13 for Windows assessed the internal structure of the YFAS-S scale. The onedimensional solution was tested, using the maximum likelihood and the robust estimator. Two CFA analyses were conducted: one for the original items and one for the generated variables that assess the eight DSM dependence criteria. Analyses were also replicated for the whole sample $(n=207)$ and for the group of ED patients $(n=125)$. Overall goodness-of-fit statistics were assessed through the root-mean-squared error of approximation (a fit was considered to be good for values lower than 0.10 ; Kline, 2010) and coefficient of determination (valuing the overall predictive capacity of the model).

Receiver operating characteristic (ROC) analyses measured the screening capacity of the YFAS-S scale scores to identify the presence of an ED and the different subtypes.

Binary logistic regressions analysed the capacity of the YFAS-S items to identify the presence of an $\mathrm{ED}$, and a $t$-test procedure compared YFAS-S dimensional score between HC and ED patients. The comparison between ED subtypes was also carried out with binary logistic models and analysis of variance (ANOVA) procedure.

Correlation coefficients valued the association between YFAS-S scale scores and clinical measures (for ED, general psychopathology and personality traits). Because of the large sample size and the consequent high statistical power, small correlations tended to achieve statistical significance, so the interpretation of $r$-coefficients was based on the own size: $|r|<.20$ poor relationship, $.20<|r|<.30$ moderate and $|r|>.30$ good.

\section{Results}

\section{Psychometric properties of the YFAS-S}

The YFAS-S binary screen score showed excellent specificity (Sp; 80 HCs met negative screen, $\mathrm{Sp}=97.6 \%, 95 \% \mathrm{CI}: 91.5$ to $99.3 \%)$ and moderate Se (91 ED patients met positive screen, $\mathrm{Se}=72.8 \%, 95 \%$ 
CI: $64.479 .8 \%$ ). The area under the ROC curve measuring the validity of the YFAS-S dimensional score to screen between HC and ED patients was excellent (area under ROC curve $($ AUC) $=0.901$; 95\% CI: 0.86 to 0.94 ). Considering DSM-5 ED subtypes, YFAS-S binary screen score obtained $\mathrm{Se}=60.0 \%$ and $\mathrm{Sp}=58.7 \%$ for $\mathrm{AN}$, $\mathrm{Se}=81.5 \%$ and $\mathrm{Sp}=68.0 \%$ for $\mathrm{BN}, \mathrm{Se}=76.9 \%$ and $\mathrm{Sp}=57.2 \%$ for $\mathrm{BED}$ and $\mathrm{Se}=72.2 \%$ and $\mathrm{Sp}=57.7 \%$ for EDNOS. The YFAS-S dimensional score achieved good AUC to discriminate the DSM-5 $\mathrm{BN}(\mathrm{AUC}=0.83)$ and BED subtypes $(\mathrm{AUC}=0.84)$.

Other psychometric evidence for the YFAS-S is available as supplementary material (Tables S1-S2). The capacity to discriminate between HC and ED patients was statistically significant for all the original YFAS-S items, except for item 19 'consuming even though emotional-physical problems' and item 24 'successful in reducing-stopping eating', as well as for the YFAS-S dimensional scores (Table S1).

Confirmatory factor analysis achieved adequate goodness of fit for the one-dimensional solution both for the original YFAS-S item codification and for the generated YFAS-S binary DSM dependence criteria (Table S2), and the only item with a nonsignificant coefficient was item 24 'successful in reducingstopping eating' for the model obtained into the ED sample. Internal consistence measured through Cronbach's alpha ranged between moderate $(\alpha=.71$, for the generated YFAS-S binary dependence scores into the ED sample) and excellent $(\alpha=.95$ for the original YFAS-S items into the whole sample).

\section{Prevalence of FA and comparison between HC and ED subtypes}

The prevalence of participants who met the criteria for the YFAS-S ' $F A$ ' was $72.8 \%$ in the ED sample and $2.4 \%$ in the HC sample $\left(\chi^{2}=99.1\right.$, d.f. $\left.=1, p<.001\right)$. Based on DSM-5 taxonomy, differences in the YFAS-S binary screen between ED subtypes were not statistically significant $\left(\chi^{2}=5.5\right.$, d.f. $\left.=3, p=.140\right): 60.0 \%$ of AN met the YFAS-S binary positive screen, $81.5 \%$ of $\mathrm{BN}, 76.9 \%$ of BED and $72.2 \%$ of EDNOS. Focusing on the AN subtypes, the YFAS-S binary screen was positive for $50 \%$ of the AN restrictive (AN-R) subtype compared with $85.7 \%$ of the AN binge-purging (AN-BP) subtype, obtaining a quasi-significant statistical result and a high effect size for the difference $\left(\chi^{2}=2.79\right.$, d.f. $=1, p=.095$; $|d|=0.83)$. The comparison of the prevalence of the YFAS-S binary positive screen between the AN-R subtype and the cluster composed by the other ED subtypes achieved a statistically significant result and a moderate effect size (50 vs $79.35 \% ; \chi^{2}=7.89$, d.f. $=1$, $p=.005 ;|d|=0.64)$.

\section{YFAS-S differences between ED subtypes}

Table 1 shows the presence of DSM dependence criteria based on the YFAS-S, among HC and ED patients. All the criteria achieved statistical differences with high effect size (significant lower prevalence for HC), except for the criterion 'use continues despite knowledge of adversity'. The mean for the YFAS-S dimensional score was also statistically different and with a high effect size comparing HC and ED patients ( 1.7 vs $4.7, t=13.1$, d.f. $=205$, $p>.001,|d|=1.96)$.

Table 2 shows the differences in the YFAS-S scores (for both the YFAS-S binary variables measuring DSM addiction criteria and the YFAS-S dimensional measure) between ED subtypes. As a whole, the prevalence for the DSM addiction criteria was lowest for the AN restricting subtype and the highest for $\mathrm{BN}, \mathrm{AN}-\mathrm{BP}$ and $\mathrm{BED}$. The DSM addiction criterion with many significant pairwise comparisons was 'substance taken in larger amount for a longer period than intended' (the prevalence was equal for EDNOS and $\mathrm{AN}$, around $30-33.3 \%$, and for $\mathrm{BN}$ and $\mathrm{BED}$, it was around $72-77 \%)$. The DSM addiction criterion 'clinical impairmentdistress' only differed between AN-BN, and the prevalence of the DSM addiction criterion 'persistent desire-attempts to quit' was near $100 \%$ in all the ED subtypes. The mean of the YFAS-S

Table 1 Prevalence of DSM dependence criteria (as measured by YFAS-S) and comparison between HC and ED patients

\begin{tabular}{|c|c|c|c|c|c|c|c|}
\hline & \multicolumn{2}{|c|}{ Prevalence $(\%)$} & \multicolumn{5}{|c|}{ Logistic regression } \\
\hline & $\mathrm{HC}$ & $\mathrm{ED}$ & $p$ & OR & & R) & $|d|$ \\
\hline \multicolumn{8}{|l|}{ YFAS-S dependence criteria (prevalence comparison) } \\
\hline Consumed more than planned & 4.88 & 53.60 & $<.001$ & 22.53 & 7.77 & 65.3 & $1.27^{\star *}$ \\
\hline Unable to cut down or stop & 84.15 & 99.20 & .003 & 23.36 & 2.99 & 182.4 & $0.57^{\star}$ \\
\hline Great deal of time spent & 10.98 & 63.20 & $<.001$ & 13.93 & 6.37 & 30.4 & $1.29^{* *}$ \\
\hline Important activities given up & 4.88 & 70.40 & $<.001$ & 46.38 & 15.82 & 136.0 & $1.84^{* *}$ \\
\hline Use despite consequences & 50.00 & 56.80 & .337 & 1.31 & 0.75 & 2.30 & 0.14 \\
\hline Tolerance & 12.20 & 64.00 & $<.001$ & 12.80 & 6.01 & 27.2 & $1.26^{\star *}$ \\
\hline Withdrawal & 7.32 & 59.20 & $<.001$ & 18.38 & 7.44 & 45.4 & $1.32^{\star *}$ \\
\hline \multirow[t]{3}{*}{ Impairment or distress } & 4.88 & 80.00 & $<.001$ & 78.00 & 26.06 & 233.4 & $2.34^{* *}$ \\
\hline & \multicolumn{2}{|c|}{ Means } & \multicolumn{5}{|c|}{$t$-test mean comparison } \\
\hline & $\mathrm{HC}$ & ED & $p$ & MD & & ID) & $|d|$ \\
\hline YFAS-S score (number of dependence criteria met) & 1.74 & 4.66 & $<.001$ & 2.92 & 2.48 & 3.36 & $1.96^{* *}$ \\
\hline
\end{tabular}

HC, healthy controls $(n=82)$; ED, eating disorder $(n=125)$; OR, odds ratio; MD, mean difference; $|d|$, Cohens'-d. Bold: significant comparison (.05 level).

${ }^{\star}$ Moderate $(|d|>.5)$ to ${ }^{* *}$ high $(|d|>.8)$ effect size. 
Table 2 Differences on YFAS-S scores between ED diagnostic subtypes

\begin{tabular}{|c|c|c|c|c|c|c|c|c|c|c|c|}
\hline \multirow[b]{3}{*}{ Diagnosis for ED: DSM-5 } & \multicolumn{4}{|c|}{ Prevalence (\%) } & \multicolumn{7}{|c|}{ Prevalence comparison (binary logistics) } \\
\hline & \multirow{2}{*}{$\begin{array}{c}\text { EDNOS } \\
1(n=18)\end{array}$} & \multirow{2}{*}{$\begin{array}{c}\text { AN } \\
2(n=40)\end{array}$} & \multirow{2}{*}{$\begin{array}{c}\mathrm{BN} \\
3(n=54)\end{array}$} & \multirow{2}{*}{$\begin{array}{c}\text { BED } \\
4(n=13)\end{array}$} & \multirow{2}{*}{$\begin{array}{l}\text { Group } \\
p\end{array}$} & \multicolumn{6}{|c|}{ Pairwise comparison: OR (odds ratio) } \\
\hline & & & & & & 2 versus 1 & 3 versus 1 & 4 versus 1 & 3 versus 2 & 4 versus 2 & 4 versus 3 \\
\hline \multicolumn{12}{|l|}{ YFAS-S symptoms (\%) } \\
\hline Consumed more than planned & 33.3 & 30.0 & 72.2 & 76.9 & $<.001$ & 0.86 & 5.20 & 6.67 & 6.07 & 7.78 & 1.28 \\
\hline Unable to cut down or stop & 100.0 & 97.5 & 100.0 & 100.0 & - & - & - & - & - & - & - \\
\hline Great deal of time spent & 66.7 & 32.5 & 79.6 & 84.6 & $<.001$ & 0.24 & 1.96 & 2.75 & 8.12 & 11.42 & 1.41 \\
\hline Important activities given up & 55.6 & 55.0 & 87.0 & 69.2 & .006 & 0.98 & 5.37 & 1.80 & 5.49 & 1.84 & 0.34 \\
\hline Use despite consequences & 44.4 & 55.0 & 55.6 & 84.6 & .203 & 1.53 & 1.56 & 6.88 & 1.02 & 4.50 & 4.40 \\
\hline Tolerance & 50.0 & 60.0 & 64.8 & 92.3 & .172 & 1.50 & 1.84 & 12.0 & 1.23 & 8.00 & 6.51 \\
\hline Withdrawal & 55.6 & 32.5 & 74.1 & 84.6 & $<.001$ & 0.39 & 2.29 & 4.40 & 5.94 & 11.42 & 1.93 \\
\hline \multirow[t]{3}{*}{ Impairment or distress } & 83.3 & 70.0 & 87.0 & 76.9 & .241 & 0.47 & 1.34 & 0.67 & 2.88 & 1.43 & 0.50 \\
\hline & \multicolumn{4}{|c|}{ Means } & Group & \multicolumn{6}{|c|}{ Pairwise comparison: mean differences (ANOVA) } \\
\hline & EDNOS & $\mathrm{AN}$ & $\mathrm{BN}$ & BED & $p$ & 2 versus 1 & 3 versus 1 & 4 versus 1 & 3 versus 2 & 4 versus 2 & 4 versus 3 \\
\hline YFAS-S diagnosis (mean) & 4.06 & 3.63 & 5.33 & 5.92 & $<.001$ & -0.43 & 1.28 & 1.87 & 1.71 & 2.30 & 0.59 \\
\hline
\end{tabular}

ED, eating disorder; EDNOS, eating disorder not otherwise specified; AN, anorexia nervosa; BN, bulimia nervosa; BED, binge eating disorder.

Bold: significant pair-wise comparison-not estimable.

dimensional score was lowest in AN, followed by EDNOS, BN and BED (pairwise comparisons were not statistically significant for anorexia-EDNOS and bulimia-binge eating).

\section{Association between YFAS-S and external clinical criteria}

Table 3 shows correlations for the YFAS-S scores and external clinical criteria, for both the ED patient sample and the total sample. As a whole, YFAS-S achieved good convergent validity compared with the EDI-2 and the SCL-90-R scores. The BMI and the number of binges per week also positively correlated with the YFAS-S dimensional score (the highest values in BMI and in the number of binges correspond to the highest YFAS-S scores).

\section{Discussion}

Besides providing a translation of the YFAS (Gearhardt et al., 2009b) into Spanish (YFAS-S) and the validation scores, this study examined the prevalence of ' $F A$ ' measured in a clinical Spanish sample of female adults diagnosed with ED according to DSM-5 criteria and assessed the differences in dependence criteria between this group and HCs. A second main objective was to explore associations between 'FA' scores, eating symptomatology and general psychopathology.

Regarding our first objective, we found that the YFAS-S had excellent $\mathrm{Sp}$ and a moderate Se when comparing ED with HC. The area under the ROC curve measuring the validity of the YFAS-S dimensional score to screen $\mathrm{HC}$ and ED patients was excellent. This result is in concordance with the psychometrical properties of the original English version of the YFAS scale (Gearhardt et al., 2009b) and suggests that this scale accurately discriminates between clinical samples with abnormal eating behaviour and healthy-eating controls.
Secondly, when comparing ED with HC, we found that $72.8 \%$ of our patients diagnosed with ED met the cut-off point of the YFAS-S for 'FA' (three or more dependence criteria and a clinically significant impairment or distress) and presented an average of 4.7 dependence symptoms. Up to now, the only ED subtypes that have been studied in relation to 'FA' were BED and $\mathrm{BN}$, with the hypothesis that 'FA' would be especially frequent among these patients. The prevalence rates found in BED range from 41.5 to 57\% (Davis et al., 2011; Gearhardt et al., 2012, Gearhardt, White et al., 2013), except in one study (Cassin \& von Ranson, 2007) whereby a prevalence of $92.4 \%$ was found. However, in this study, a standardized measure of addictive behaviours towards food was not used. In a study with women with current or remitted BN, 100\% of patients with current $\mathrm{BN}$ met 'FA' diagnosis, while only $30 \%$ of women with remitted BN did (Meule, von Rezori, \& Blechert, 2014).

The prevalence rate of the YFAS-S 'FA' in the Spanish HCs of our study was just $2.4 \%$ (with a mean of 1.7 dependence criteria), and this group did not present any general psychopathology. The preliminary validation of the YFAS (Gearhardt et al., 2009b) showed that $11.6 \%$ of their representative sample of an undergraduate population met the 'FA' criteria, and in a German validation study predominantly conducted with students, $8.8 \%$ of the participants fulfilled these criteria (Meule, Vögele, \& Kübler, 2012). A lack of an accurate psychopathological screening in previous studies may explain the discrepancy in the obtained results, as well as the exclusion, in our study, of HCs with a lifetime diagnosis of ED or a current diagnosis of any substance or non-substance abuse disorder.

Thirdly, when considering the specific dependence criteria, the overall prevalence was much higher in ED than in HCs, except for the criterion 'continued use despite negative consequences', which did not reach a significant difference, and the criterion 'unable to cut down or stop', where the difference was significant but smaller. Moreover, patients diagnosed with ED experienced 
Table 3 Correlation between YFAS-S scores and ED and psychopathological symptoms

\begin{tabular}{|c|c|c|c|c|}
\hline \multirow[b]{2}{*}{ YFAS-S scores } & \multicolumn{2}{|c|}{ ED patients $(n=125)$} & \multicolumn{2}{|c|}{ Total sample $(n=207)$} \\
\hline & Dimensional $^{\dagger}$ & Binary $^{\ddagger}$ & Dimensional $^{\dagger}$ & Binary $^{\ddagger}$ \\
\hline EDI-2: drive for thinness & $.421^{\star}$ & $.478^{\star}$ & $.649^{*}$ & $.701^{*}$ \\
\hline EDI-2: body dissatisfaction & .295 & .288 & $.584^{\star}$ & $.587^{\star}$ \\
\hline EDI-2: interoceptive awareness & $.407^{\star}$ & $.409^{*}$ & $.630^{*}$ & $.651^{\star}$ \\
\hline EDI-2: bulimia & $.553^{\star}$ & $.420^{\star}$ & $.670^{*}$ & $.612^{*}$ \\
\hline EDI-2: interpersonal distrust & .246 & .255 & $.537^{\star}$ & $.554^{*}$ \\
\hline EDI-2: ineffectiveness & .142 & .099 & $.344^{*}$ & $.322^{\star}$ \\
\hline EDI-2: maturity fears & .265 & $.341^{\star}$ & $.397^{\star}$ & $.462^{*}$ \\
\hline EDI-2: perfectionism & .203 & .291 & $.436^{\star}$ & $.500^{*}$ \\
\hline EDI-2: impulse regulation & .253 & $.323^{\star}$ & $.493^{\star}$ & $.559^{\star}$ \\
\hline EDI-2: asceticism & .180 & .134 & $.454^{*}$ & $.450^{*}$ \\
\hline EDI-2: social insecurity & .141 & .011 & .029 & -.017 \\
\hline EDI-2: total score & $.397^{\star}$ & $.391^{\star}$ & $.638^{\star}$ & $.658^{\star}$ \\
\hline SCL-90-R: somatisation & .246 & .198 & $.506^{\star}$ & $.492^{*}$ \\
\hline SCL-90-R: obsessive/compulsive & $.303^{\star}$ & .186 & $.530^{\star}$ & $.479^{*}$ \\
\hline SCL-90-R: interpersonal sensitivity & $.343^{\star}$ & .261 & $.565^{\star}$ & $.555^{\star}$ \\
\hline SCL-90-R: depressive & $.364^{\star}$ & .286 & $.618^{\star}$ & $.597^{\star}$ \\
\hline SCL-90-R: anxiety & .253 & .257 & $.491^{\star}$ & $.502^{\star}$ \\
\hline SCL-90-R: hostility & .211 & .133 & $.475^{\star}$ & $.432^{*}$ \\
\hline SCL-90-R: phobic anxiety & .276 & .217 & $.479^{*}$ & $.465^{\star}$ \\
\hline SCL-90-R: paranoid ideation & .198 & .204 & $.452^{\star}$ & $.477^{\star}$ \\
\hline SCL-90-R: psychotic & .292 & .255 & $.555^{\star}$ & $.554^{\star}$ \\
\hline SCL-90-R: GSI score & $.346^{*}$ & .285 & $.600^{*}$ & $.588^{\star}$ \\
\hline SCL-90-R: PST score & $.324^{\star}$ & .229 & $.538^{\star}$ & $.515^{\star}$ \\
\hline SCL-90-R: PSDI score & $.354^{\star}$ & $.328^{\star}$ & $.613^{\star}$ & $.607^{\star}$ \\
\hline BMI (present) & $.398^{\star}$ & .153 & $.368^{*}$ & .196 \\
\hline Age (years old) & .142 & .125 & .275 & .283 \\
\hline Binges per week & $.452^{\star}$ & .253 & - & - \\
\hline Vomits per week & .216 & .124 & - & - \\
\hline Laxatives per week & .091 & .101 & - & - \\
\hline Onset (years) & .137 & .128 & - & - \\
\hline Evolution (years) & .138 & .111 & - & - \\
\hline Number of treatments & -.195 & -.122 & - & - \\
\hline
\end{tabular}

YFAS-S dimensional: total of DSM dependence criteria. YFAS-S binary: positive vs negative classification of 'FA'.

${ }^{\dagger}$ Pearson correlation.

${ }^{\ddagger}$ Biserial point correlation.

${ }^{\star}$ Good effect size $(|r| \geq .30)$.

— indicates that the measure was only available for ED patients.

significantly more impairment or distress because of 'FA' symptoms than the HC. Comparison of 'FA' symptoms between ED diagnostic subtypes showed significant differences in all dependence criteria except for 'unable to cut down or stop'. In contrast to more restrictive or purge-only disorders (AN-R subtype and EDNOS), patients diagnosed with EDs in which binges are frequent (namely $\mathrm{AN}-\mathrm{BP}$ subtype, $\mathrm{BN}$ and $\mathrm{BED}$ ) tend to consume more high-fat/high-sugar caloric food than planned, are more likely to give up important activities and experience more withdrawal symptoms. They therefore present a higher number of dependence symptoms. This is consistent with the other obtained results based on the 'FA' category, where AN-BP subtype showed the highest scores (85.7\%), followed by BN and BED (81.5 and
76.9\% respectively), whereas EDNOS and the AN-R subtype displayed the lowest scores (72.2 and 50\% respectively). Paradoxically, half of the patients who presented more restrictive features (AN-R) also showed positive 'FA' scores. This result suggests that, although patients with the AN-R subtype tend to show more restrictive eating behaviours in order to suppress impulses of hunger and a loss of control over food intake (Witt \& Lowe, 2014), they may nonetheless experience an internal subjective impulse for disinhibition and a craving for food or hedonic eating. Individuals with AN who exhibit more hedonic hunger for food are also more prone to weight gain (Witt \& Lowe, 2014) and may potentially be more likely to exhibit signs of addictive-like eating. These patients' fears, and the accompanying cognitive distortions about food and weight, may also influence their subjective impression of their abnormal eating behaviour and what they consider as excessive food intake (Gendall, Sullivan, Joyce, \& Bulik, 1997). The extent to which these patients are more likely to present a diagnostic cross over along the course of their ED (becoming AN-BP or BN over the years) could not be answered in this study but should be assessed in the future. It has been previously proposed (Eddy et al., 2008) that $57.5 \%$ of patients with a diagnosis of the AN restricting subtype cross over to the AN-BP subtype or to BN during follow-up (55\% to the AN-BP subtype and $10 \%$ to $\mathrm{BN}$ ), which makes the possible involvement of a subjective 'FA' of clinical importance. Therefore, in future studies, there are two aspects that may have to be taken into account. It may be useful to analyse the potential relevance of subjective disinhibition towards food in the AN-R subtype for the cross over to other binge-related EDs over the course of the disorder.

Fourthly, regarding our final objective, we found that the YFAS-S dimensional/binary scores of the ED patients were positively associated with higher eating psychopathology and ED severity. Thus, the presence of addiction-related symptoms seems to be a sign of more severe eating pathology among ED patients. In contrast to previous research (Davis et al., 2011; Eichen et al., 2013), we found an association between BMI and YFAS-S symptom scores, but this association was not significant when the classification of ' $F A$ ' was considered, as recently described (Burgess et al., 2014). As suggested in previous studies, BMI may be a consequence of disinhibited eating behaviour rather than a factor related to 'FA' (Villarejo et al., 2014). Moreover, higher dimensional scores were also associated with the number of binges per week but not with the number of vomits or use of laxatives per week. This finding supports the hypotheses that the YFAS should be associated with binge eating and not necessarily to purging episodes and that the YFAS may not predict disordered eating behaviour indiscriminately but is specifically linked to disinhibited eating (Burgess et al., 2014). The development of tolerance is a characteristic of addictive behaviours; in our sample, $64 \%$ of ED patients meet the YFAS-S tolerance criteria, in comparison with only $12 \%$ of the HCs. In our study, differences in tolerance among ED subtypes reached significance when comparing binging ED subtypes with restrictive ED subtypes. As other authors have suggested (Burmeister, Hinman, Koball, Hoffmann, \& Carels, 2013), higher tolerance and withdrawal symptoms are linked to binge symptomatology and to poorer therapy response.

We did not find an association between 'FA' scores and the age of onset of the disorder, its duration, or with the number of treatments. 
Furthermore, higher scores on the YFAS-S and a 'FA' classification were associated with a higher general psychopathology (namely depressive and obsessive-compulsive symptomatology, as well as interpersonal Se). These results are in accordance with studies conducted with patients with BED, which found that more addiction-related symptoms were associated with higher levels of negative affect and more severe eating pathology (Gearhardt et al., 2012, Gearhardt, White et al., 2013; Meule et al., 2014). Furthermore, research has recently shown that emotional eating, especially in response to negative emotions, significantly moderates the relationship between mood and food intake (Cebolla, Barrada, van Strien, Oliver, \& Baños, 2014; Van Strien et al., 2013; Zhu et al., 2012; Espeset et al., 2012).

\section{Limitations}

Although the current study addresses a number of gaps in the literature, there are limitations to consider. First, the current study was conducted solely with female participants because of the small number of male ED patients. Thus, it is unclear whether these findings could be generalized to men. Secondly, compared with the AN and $\mathrm{BN}$ groups, the BED group was rather small $(n=13)$, which could have hidden some interesting findings, and the $\mathrm{HC}$ sample was slightly younger than the clinical groups. Finally, the study is cross sectional; therefore, it is unclear whether addictive-like eating behaviour is a cause, correlate or consequence of disordered eating.

An important step for the future is to conduct longitudinal analyses on the association between addictive-like eating and disordered eating. Of special importance is the assessment of whether higher scores on the YFAS are predictive of differential responses to ED treatments. Furthermore, as recently suggested with regard to the concept of hedonic eating (Witt \& Lowe,
2014), the food addiction construct should be further analysed to understand the loss of control over eating in AN and its potential link to the reported diagnostic cross over from the AN-R subtype to the AN-BP subtype, as it has been previously described (Eddy et al., 2008). Research in addictive-like eating patterns also needs to be extended to samples of emotional eaters and to samples of people with substance or non-substance abuse disorder, as well as to general population samples.

\section{Conclusions}

The results of this study support the good psychometrical properties (internal structure and discriminative capacity to differentiate between ED and controls) of the Spanish version of the YFAS. Higher YFAS-S scores are associated with the bingeing ED subtype (namely AN-BP, BN and BED), with more severe eating pathology and overall psychopathology (namely negative affect and depression) and a greater BMI. Although the 'FA' construct is able to differentiate between $\mathrm{ED}$ and $\mathrm{HC}$, it needs to be further explored and tested to understand its implication in the diagnostic cross over in $\mathrm{AN}$ and its predictive capacity regarding response to treatment.

\section{Acknowledgements}

Financial support was received from Fondo de Investigación Sanitaria (PI11/210). Centro de Investigación Biomédica en Red (CIBER) Fisiopatología de la Obesidad y Nutrición, CIBER Diabetes y Enfermedades Metabólicas and CIBER Salud Mental are initiatives of Instituto de Salud Carlos III. The authors declare no conflict of interest.

\section{REFERENCES}

APA. (2000). Diagnostic and Statistical Manual of Mental Disorders: DSM-IV-TR. Washington, DC: American Psychiatric Association.

APA. (2013). Diagnostic and statistical manual of mental disorders: DSM-5. Washington, DC: American Psychiatric Association.

Avena, N. M. (2010). The study of food addiction using animal models of binge eating. Appetite, 55, 734-737.

Avena, N. M., Bocarsly, M. E., \& Hoebel, B. G. (2012). Animal models of sugar and fat bingeing: relationship to food addiction and increased body weight. Methods in Molecular Biology, 829, 351-365.

Avena, N. M., Rada, P., \& Hoebel, B. G. (2008). Evidence for sugar addiction: behavioral and neurochemical effects of intermittent, excessive sugar intake. Neuroscience and Biobehavioral Reviews, 32, 20-39.

Brislin, R. W. (1970). Back-Translation for Cross-Cultural Research. Journal of Cross-Cultural Psychology, 1, 185-216.

Burgess, E. E., Turan, B., Lokken, K. L., Morse, A., \& Boggiano, M. M. (2014). Profiling motives behind hedonic eating. Preliminary validation of the Palatable Eating Motives Scale. Appetite, 72, 66-72.

Burmeister, J. M., Hinman, N., Koball, A., Hoffmann, D. A., \& Carels, R. A. (2013). Food addiction in adults seeking weight loss treatment. Implications for psychosocial health and weight loss. Appetite, 60, 103-110.
Calero-Elvira, A., Krug, I., Davis, K., López, C., Fernández-Aranda, F., \& Treasure, J. (2009). Meta-analysis on drugs in people with eating disorders. European Eating Disorders Review, 17, 243-259.

Cassin, S. E., \& von Ranson, K. M. (2007). Is binge eating experienced as an addiction? Appetite, 49, 687-690.

Cebolla, A., Barrada, J. R., van Strien, T., Oliver, E., \& Baños, R. (2014). Validation of the Dutch Eating Behavior Questionnaire (DEBQ) in a sample of Spanish women. Appetite, 73, 58-64.

Clark, S. M., \& Saules, K. K. (2013). Validation of the Yale Food Addiction Scale among a weight-loss surgery population. Eating Behaviors, 14, 216-219.

Davis, C., Curtis, C., Levitan, R. D., Carter, J. C., Kaplan, A. S., \& Kennedy, J. L. (2011). Evidence that "food addiction" is a valid phenotype of obesity. Appetite, 57, 711-717.

Davis, C., Loxton, N. J., Levitan, R. D., Kaplan, A. S., Carter, J. C., \& Kennedy, J. L. (2013). "Food addiction" and its association with a dopaminergic multilocus genetic profile. Physiology and Behavior, 118, 63-69.

Derogatis, L. R. (1990). SCL-90-R. Administration, scoring and procedures manual. Baltimore, MD: Clinical Psychometric Research.

Derogatis, L. (2002). SCL-90-R. Cuestionario de 90 sintomas- Manual. Madrid: TEA.

Eddy, K. T., Dorer, D. J., Franko, D. L., Tahilani, K., ThompsonBrenner, H., \& Herzog, D. B. (2008). Diagnostic Crossover in Anorexia Nervosa and Bulimia Nervosa: Implications for DSMV. The American Journal of Psychiatry, 165, 245-250.
Eichen, D. M., Lent, M. R., Goldbacher, E., \& Foster, G. D. (2013). Exploration of "food addiction" in overweight and obese treatment-seeking adults. Appetite, 67, 22-24.

Espeset, E. M. S., Gulliksen, K. S., Nordbø, R. H. S., Skårderud, F. \& Holte, A. (2012). The Link Between Negative Emotions and Eating Disorder Behaviour in Patients with Anorexia Nervosa. European Eating Disorders Review, 20, 451-460.

Fernandez-Aranda, F., \& Turon, V. (1998). Trastornos de la alimentación. Barcelona: Masson.

First, M., Gibbon, M., Spitzer, R., \& Williams, J. (1996). Users guide for the structured clinical interview for DSM IV Axis I disordersResearch version (SCID-I, Version 2.0). New York: New York State Psychiatric Institute.

Garner, D. M. (1991). Eating disorder inventory-2. Odessa: Psychological Assessment Resources.

Garner, D. M. (1998). Inventario de Trastornos de la Conducto Alimentaria (EDI-2)-Manual. Madrid: TEA.

Gearhardt, A., Corbin, W., \& Brownell, K. (2009a). Food addiction: an examination of the diagnostic criteria for dependence. Journal of Addiction Medicine, 3, 1-7.

Gearhardt, A., Corbin, W., \& Brownell, K. (2009b). Preliminary validation of the Yale Food Addiction Scale. Appetite, 52, 430-436.

Gearhardt, A., Roberto, C., Seamans, M., Corbin, W., \& Brownell, K. (2013). Preliminary validation of the Yale Food Addiction Scale for children. Eating Behaviors, 14, 508-512.

Gearhardt, A., White, M., Masheb, R., \& Grilo, C. (2013). An examination of food addiction in a racially diverse sample of obese 
patients with binge eating disorder in primary care settings. Comprehensive Psychiatry, 54, 500-505.

Gearhardt, A., White, M., Masheb, R., Morgan, P., Crosby, R., \& Grilo, C. (2012). An examination of the food addiction construct in obese patients with binge eating disorder. International Journal of Eating Disorders, 45, 657-663.

Gearhardt, A., Yokum, S., Orr, P., Stice, E., Corbin, W., \& Brownell, K. (2011). Neural correlates of food addiction. Archives of General Psychiatry, 68, 808-816.

Gendall, K. A., Sullivan, P. F., Joyce, P. R., \& Bulik, C. M. (1997). Food cravings in women with a history of anorexia nervosa. The International Journal of Eating Disorders, 22, 403-409.

Goldberg, D. P., \& Hillier, V. F. (1979). A scaled version of the General Health Questionnaire. Psychological Medicine, 9, 139-145. doi: 10.1017/S0033291700021644.

Hone-Blanchet, A., \& Fecteau, S. (2014). Overlap of food addiction and substance use disorders definitions: Analysis of animal and human studies. Neuropharmacology. doi: 10.1016/j. neuropharm.2014.05.019.

Jiménez-Murcia, S., Steiger, H., Isräel, M., Granero, R., Prat, R., Santamaría, J. J., et al. (2013). Pathological gambling in eating disorders: prevalence and clinical implications. Comprehensive Psychiatry, 54, 1053-1060. doi: 10.1016/j.comppsych.2013.04.014.

Johnson, P. M., \& Kenny, P. J. (2010). Dopamine D2 receptors in addiction-like reward dysfunction and compulsive eating in obese rats. Nature Neuroscience, 13, 635-641.
Kline, R. B. (2010). Principles and practice of structural equation modeling (3rd ed.). New York, New York: Guilford Press.

Lobo, A., Pérez-Echeverría, M. J., \& Artal, J. (2009). Validity of the scaled version of the General Health Questionnaire (GHQ-28) in a Spanish population. Psychological Medicine, 16, 135-140. doi: $10.1017 /$ S0033291700002579.

Machado, P. P., Goncalves, S., \& Hoek, H. W. (2013). DSM-5 reduces the proportion of EDNOS cases: evidence from community samples. International Journal of Eating Disorders, 46, 60-65.

Meule, A., Heckel, D., \& Kübler, A. (2012). Factor structure and item analysis of the Yale Food Addiction Scale in obese candidates for bariatric surgery. European Eating Disorders Review, 20, 419-422.

Meule, A., Vögele, C., \& Kübler, A. (2012). Deutsche Übersetzung und Validierung der Yale Food Addiction Scale. Diagnostica, 58, 115-126. doi: 10.1026/0012-1924/a000047.

Meule, A., von Rezori, V., \& Blechert, J. (2014). Food addiction and bulimia nervosa. European Eating Disorders Review, 22, 331-337. Nogueiras, R., Romero-Picó, A., Vazquez, M. J., Novelle, M. G., López, M., \& Diéguez, C. (2012). The Opioid System and Food Intake: Homeostatic and Hedonic Mechanisms. Obesity Facts, 5, 196-207.

Speranza, M., Revah-Levy, A., Giquel, L., Loas, G., Venice, J.-L \& Jeammet, P. et al. (2012). An Investigation of Goodman's Addictive Disorder Criteria in Eating Disorders. European Eating Disorders Review, 20, 182-189.

Van Strien, T., Cebolla, A., Etchemendy, E., Gutiérrez-Maldonado, J., Ferrer-García, M., Botella, C., et al. (2013). Emotional eating and food intake after sadness and joy. Appetite, 66, 20-25.
Villarejo, C., Jiménez-Murcia, S., Álvarez-Moya, E., Granero, R., Penelo, E., Treasure, J., et al. (2014). Loss of control over eating: a description of the eating disorder/obesity spectrum in women. European Eating Disorders Review, 22, 25-31.

Volkow, N. D., Wang, G. J., Fowler, J. S., \& Telang, F. (2008). Overlapping neuronal circuits in addiction and obesity: evidence of system pathology. Philosophical Transactions of the Royal Society of London, Series B: Biological Sciences, 363, 3191-3200.

Volkow, N. D., Wang, G. J., Fowler, J. S., Tomasi, D., \& Baler, R. (2012). Food and drug reward: overlapping circuits in human obesity and addiction. Current Topics in Behavioral Neurosciences, $11,1-24$.

Wang, G. J., Volkow, N. D., Thanos, P. K., \& Fowler, J. S. (2009). Imaging of brain dopamine pathways: implications for understanding obesity. Journal of Addiction Medicine, 3, 8-18.

Witt, A., \& Lowe, M. R. (2014). Hedonic hunger and binge eating among women with eating disorders. The International Journal of Eating Disorders, 47, 273-280.

Ziauddeen, H., \& Fletcher, P. C. (2013). Is food addiction a valid and useful concept? Obesity Reviews: An Official Journal of the International Association for the Study of Obesity, 14, 19-28.

Zhu, Y., Hu, X., Wang, J., Chen, J., Guo, Q., \& Li, C. et al. (2012). Processing of Food, Body and Emotional Stimuli in Anorexia Nervosa: A Systematic Review and Meta-analysis of Functional Magnetic Resonance Imaging Studies. European Eating Disorders Review, 20, 439-450.

\section{Supporting information}

Additional supporting information may be found in the online version of this article at publisher's web site. 